\title{
Zur Aktualität der Jung'schen Analytischen Psychologie
}

\section{Theodor Seifert}

Online publiziert: 24. Mai 2014

(C) Springer-Verlag Berlin Heidelberg 2014

Carl Gustav Jung war ein Visionär. Er hatte die Fähigkeit in dem, was er sah - sowohl bei seinen Patienten als auch in den alten Schriften, die er studierte - die Muster zu erkennen, die dem Gesehenen zugrunde liegen. Weil diese Begabung für den Erkenntnisprozess von großer Bedeutung ist, zeichnet sie all die Menschen aus, die in sich den Ruf des Schöpferischen hören und die Neugier und Kraft aufbringen, ihm zu folgen. ,Wäre die Bewegtheit und das Schillern der Seele nicht, der Mensch würde in seiner größten Leidenschaft, der Trägheit, zum Stillstand kommen“, meinte Jung (G. W. 9/1, §56).

Mir bedeutet die Jung'sche Psychologie seit Jahrzehnten sehr viel, denn ich fand zu Beginn meines Studiums am C.G. Jung-Institut in Zürich eine vertraute, damals noch kaum formulierbare geistige Welt, die sich dann im Laufe der Studien, der persönlichen Erlebnisse in Lehranalyse und Weiterbildung als eine wirkliche geistige Heimat erwies, die sie bis heute geblieben ist. Am Jung'schen Werk ist für mich faszinierend, dass ich dieselben Arbeiten nach vielen Jahren immer wieder lesen kann und Neues entdecke, was mir seinerzeit, aus welchen Gründen auch immer, noch nicht aufgefallen war. In jedem Fall bietet sie eine Weite an Perspektiven und einen Reichtum an Gesichtspunkten, Erfahrungen und theoretischen Folgerungen, die ich in diesem Umfang sonst im Rahmen meines Faches nirgends gefunden habe. Insofern betrachte ich die analytische Psychologie als allzeit aktuell.

Jung hatte sich der Weite des damaligen Wissenschaftsbereichs angeschlossen und Kontakt zu vielen bedeutenden Vertretern anderer Fachrichtungen geknüpft. Es sollen hier nur einige erwähnt werden: Karl Kerenyi, der ihn zum Studium der Mytholo-

Dr. rer. biol. hum. T. Seifert $(\bowtie)$

Mörikestr. 54, 71299 Wimsheim, Deutschland

E-Mail: asibrd@t-online.de 
gie, insbesondere der griechischen anregte; der Basler Biologe Adolf Portmann, dem er den Blick auf die Bedeutung der Bilder in der lebendigen Energieumwandlung verdankt, und der Sinologe Richard Wilhelm, über den er Zugang zur Welt des Ostens fand. Er schrieb die Einleitung zu Wilhelms Geheimnis der Goldenen Blüte, einem alten chinesischen alchemistischen Text. Sie ist für mich bis heute eine der besten Einführungen in das Jung'sche Werk.

Über seine alchemistischen Studien gibt es Beziehungen zur Wissenschaftsgeschichte, aber auch zur Erkenntnis- und Wissenschaftstheorie - zum Beispiel in seiner Arbeit zu den theoretischen Überlegungen zum Wesen des Psychischen, das ein gundlegendes Werk ist, welches man nicht oft genug konsultieren kann. Die immer wieder diskutierte Frage: „Was ist Zeit?“ findet in seiner Auseinandersetzung mit Vergangenheit, Gegenwart und Zukunft besondere Berücksichtigung. Das Aktualkonfliktmodell, das Jung schon 1912 veröffentlichte, stellt die Frage der Gegenwart und des später so genannten Hic et nunc ganz in den Mittelpunkt, denn nur in der Gegenwart liegt das aktuelle Problem. Die Ich-Funktionen und auch die prospektive Funktion, die Zukunftsfähigkeit der Psyche und die damit angedeuteten Möglichkeiten der Wandlungen und Neukonstellationen des Archetypus gehören ebenfalls hierher. Die große Thematik der inneren Führung des Menschen ist wesentlich in der praktischen Arbeit der analytischen Psychologie; insbesondere hat sich für mich hier die Beachtung und Hinzuziehung der aktiven Imagination, der Träume, Fantasien und Symbole bewährt.

Im vorliegenden Heft des Forum der Psychoanalyse schildert Verena Kast die Arbeit mit einer Patientin, die aufgrund früher dysfunktionaler Beziehungsmuster unter Albträumen litt. Wie im Rahmen einer aktiven Imagination und im Schutz der therapeutischen Beziehung die Berührung mit den tieferen, bisher unbewusst gebliebenen Seelenschichten der Patientin und deren Integration in das bewusste Erleben gelang, wird hier sehr anschaulich, ja äußerst spannend geschildert. Jung war von der Methode der aktiven Imagination so überzeugt, dass er schrieb: „Auf diese Weise können Sie nicht nur Ihr Unbewusstes analysieren, sondern Sie geben dem Unbewussten eine Chance, Sie zu analysieren. Und so erschaffen Sie nach und nach die Einheit von Bewusstsein und Unbewusstem, ohne die es überhaupt keine Individuation

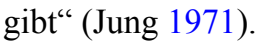

Nicht zu vergessen ist das Heilungsmodell mit durchaus philosophischen Implikationen im Hinblick auf seine Grundüberzeugung, dass seine Psychologie eine dialektische Psychologie ist. Die Dynamik der Polaritäten, des Gegensatzprinzips, gehört hierher, aber auch die für mein Verständnis recht wenig beobachtete bzw. erwähnte Dynamik des Archetyps der ,conjunctio“ dürfte hier die Theorie unseres psychotherapeutischen Handelns noch wesentlich erweitern.

Die Beziehung von Bewusstem und Unbewusstem, aber auch die von Mensch zu Mensch und vor allem die, welche Gegensätzliches miteinander verbindet, war für Jung so bedeutsam, dass er sein letztes großes wissenschaftliches Werk ,Mysterium coniunctionis“ nannte. Um Beziehungen geht es auch in dem Beitrag von $\mathrm{C}$. Maier. Diese sind hier geheimnisvolle, sehr subtile, äußere und innere Verflechtungen zwischen dem Psychoanalytiker Wilfred Bion und seinem Analysanden, dem Nobelpreisträger für Literatur, Samuel Beckett, um die Idee von ,imaginary twins“ und schließlich dem Problem der Kryptomnesie. Wie C.G. Jung aufgrund seiner 
„Tavistock Lectures” zum „Kristallisationspunkt“ zwischen Bion und Beckett wurde, beschreibt der Autor in faszinierender Weise.

Sie führt direkt zum „Herzstück“ der analytischen Psychologie, zum Archetypenkonzept. Es ist im Beitrag von Christian Roesler und Milena Sotirova-Kohli sehr differenziert, profund und ausführlich dargestellt.

In diesem Zusammenhang ist die Verbindung zwischen Jung und dem Nobelpreisträger für Physik, Wolfgang Pauli, hervorzuheben. Sie war insofern schicksalhaft, als Pauli aufgrund seines genialen introvertierten Denkens große Probleme mit der äußeren Wirklichkeit hatte - fast wäre er in alkoholischen Exzessen verwahrlost und Jung mit der Bitte um eine Psychoanalyse aufsuchte. Doch dieser verwies ihn an eine seiner Schülerinnen, denn er wollte Pauli als Gesprächspartner erhalten, um mit ihm die großen Themen der Komplementarität und Synchronizität zu untersuchen. Viele Physiker hatten sich damals auf die Suche nach der geheimnisvollen Zahl 137 begeben. Wolfgang Pauli ist in seinem 59. Lebensjahr überraschend an Pankreaskrebs gestorben - in einem Zürcher Spital, im Zimmer 137.

Diese Beziehung ist in dem äußerst lesenswerten Buch von Arthur I. Miller (2011) 137 - C. G. Jung, Wolfgang Pauli und die Suche nach der kosmischen Zahl dokumentiert. Es ist auch eines über die Zeitgeschichte der Quantenphysik des vergangenen Jahrhunderts und über C.G. Jungs Ideen zum kollektiven Unbewussten, zu den Archetypen und letztlich zur Idee des „unus mundus“. Für mein Verständnis hat Jung hiermit ein umfassendes Konzept vorgelegt. Es stellt die Entwicklung des Menschen, seine Stellung in der Welt und seine Verbindung zum All-Einen dar.

Heute zeigt sich der unus mundus als World Wide Web, das in den 1990er Jahren von dem Briten Tim Berners-Lee an der Forschungseinrichtung Conseil Européen pour la Recherche Nucléaire (CERN) bei Genf entwickelt wurde, an dessen Gründung übrigens Wolfgang Pauli beteiligt war. Wenn man sich im Internet die Bilder vom Large Hadron Collider anschaut, in dem am CERN das letzte Teilchen zur Formulierung der Weltformel gesucht wird, kommt man nicht umhin, Parallelen zu den Darstellungen eines Mandalas oder eines Kirchenfensters zu sehen. Jung wies in seinem Werk an vielen Stellen auf den Archetyp des Mandalas als Symbol für das alles umfassende Selbst hin, und für Bion war das O, „the ultimate reality”.

Die Zeiten ändern sich und mit ihnen die Formen und Verhaltensweisen der Menschen. Doch die Muster, aus denen die Welt sich weiter und weiter entfaltet, sind ursprünglich in sie hineingewoben. Die Welt kann vermessen und immer wieder neu ausgestaltet, das Wesen des Psychischen kann entdeckt und verstanden werden - ob mit Freud, Jung, Bion und den vielen anderen, die sich darum bemühen. Letzten Endes wird jeder, der das tut, über die Bewegtheit und das Schillern der Seele staunen und der Trägheit entsagen.

\section{Literatur}

Jung CG (1971) Gesammelte Werke. Walter, Olten Freiburg i. Br., Bd 9/1, §56

Jung CG (1971) Briefe II. Walter, Olten/Freiburg i. Br. 1971, S 92-93

Miller AI (2011) 137 - C.G. Jung, Wolfgang Pauli und die Suche nach der kosmischen Zahl. Deutsche Verlags-Anstalt, München 
Theodor Seifert, Diplompsychologe Dr. rer. biol. hum., Jg. 1931, ist seit Gründung dieser Zeitschrift Mitherausgeber bzw. Beirat und vertritt hier die Jung'sche Analytische Psychologie. Lehranalytiker und Dozent am C.G. Jung-Institut in Stuttgart und in eigener psychotherapeutischer Praxis. Seine Themen der Analytischen Psychologie sind Synchronizität, aktive Imagination, Zahl und Zeit, Mystik, Meditation und östliche Geisteshaltung. 